\title{
Disjoint unions of complete graphs characterized by their Laplacian spectrum *
}

\author{
Romain Boulet ${ }^{\dagger}$
}

Dec. 2009

\begin{abstract}
A disjoint union of complete graphs is in general not determined by its Laplacian spectrum. We show in this paper that if we only consider the family of graphs without isolated vertex then a disjoint union of complete graphs is determined by its Laplacian spectrum within this family. Moreover we show that the disjoint union of two complete graphs with $a$ and $b$ vertices, $\frac{a}{b}>\frac{5}{3}$ and $b>1$ is determined by its Laplacian spectrum. A counter-example is given when $\frac{a}{b}=\frac{5}{3}$.

Keywords: Graphs, Laplacian, complete graphs, graphs determined by its spectrum, strongly regular graphs.

AMS subject classifications: $05 \mathrm{C} 50,68 \mathrm{R} 10$.
\end{abstract}

\section{Introduction and basic results}

The Laplacian of a graph $G$ is the matrix $L$ defined by $L=D-A$ where $D$ is the diagonal matrix of the degrees of $G$ and $A$ is the adjacency matrix of $G$. The Laplacian spectrum gives some informations about the structure of the graph but determining graphs characterized by their Laplacian spectrum remains a difficult problem [2].

In this paper we focus on the disjoint union of complete graphs. A complete graph on $n$ vertices is denoted by $K_{n}$ and the disjoint union of the graphs $G$ and $G^{\prime}$ is denoted by $G \cup G^{\prime}$. The Laplacian spectrum of $K_{k_{1}} \cup K_{k_{2}} \cup \ldots \cup K_{k_{n}}$ is

$$
\left\{k_{1}^{\left(k_{1}-1\right)}, k_{2}^{\left(k_{2}-1\right)}, \ldots, k_{n}^{\left(k_{1}-1\right)}, 0^{(n)}\right\}
$$

but in general the converse is not true: a disjoint union of complete graphs is not in general determined by its Laplacian spectrum. For instance 2 the disjoint union of the Petersen graph with 5 isolated vertices is $L$-cospectral with the disjoint union of the complete graph with five vertices and five complete graphs with two vertices, these graphs are depicted in figure 1.

In this paper we show in Section 2 that the disjoint union of complete graphs without isolated vertex is determined by its Laplacian spectrum in the family of graphs without isolated vertex. Then in Section 3 we study the disjoint union

*Paper published in Electronic Journal of Linear Algebra, Volume 18, pp. 773-783, December 2009, http://www.emis.de/journals/ELA/ela-articles/articles/vol18_pp773-783.pdf

†Institut de Mathématiques de Toulouse, Université de Toulouse et CNRS (UMR 5219), France (boulet@univ-tlse2.fr). 

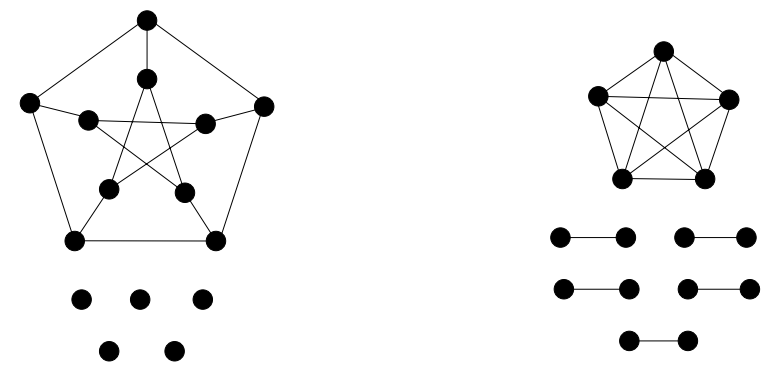

Figure 1: The graph drawn on the left is a graph non-isomorphic to a disjoint union of complete graphs but Laplacian cospectral with the disjoint union of complete graphs drawn on the right

of two complete graphs $K_{a}$ and $K_{b}$ and show that if $\frac{a}{b}>\frac{5}{3}$ then $K_{a} \cup K_{b}$ is determined by its Laplacian spectrum.

To fix notations, the set of vertices of a graph $G$ is denoted by $V(G)$ and the set of edges is denoted by $E(G)$; for $v \in V(G), d(v)$ denotes the degree of $v$. The complement of a graph $G$ is denoted by $\bar{G}$ and concerning the spectrum, $\operatorname{Sp}(G)=\left\{\mu_{1}^{\left(m_{1}\right)}, \cdots \mu_{k}^{\left(m_{k}\right)}\right\}$ means that $\mu_{i}$ is $m_{i}$ times an eigenvalue of $L$ (the multiplicity of $\mu_{i}$ is at least $m_{i}$, we may allow $\mu_{i}=\mu_{j}$ for $i \neq j$ ).

We end this introduction with some known results about the Laplacian spectrum and strongly regular graphs.

Theorem 1 [6] The multiplicity of the Laplacian eigenvalue 0 is the number of connected components of the graph.

Theorem 2 [4, 6] Let $G$ be a graph on $n$ vertices whose Laplacian spectrum is $\mu_{1} \geq \mu_{2} \geq \ldots \geq \mu_{n-1} \geq \mu_{n}=0$. Then:

1. $\mu_{n-1} \leq \frac{n}{n-1} \min \{d(v), v \in V(G)\}$.

2. If $G$ is not a complete graph then $\mu_{n-1} \leq \min \{d(v), v \in V(G)\}$.

3. $\mu_{1} \leq \max \{d(u)+d(v), u v \in E(G)\}$.

4. $\mu_{1} \leq n$.

5. $\sum_{i} \mu_{i}=2|E(G)|$.

6. $\mu_{1} \geq \frac{n}{n-1} \max \{d(v), v \in V(G)\}>\max \{d(v), v \in V(G)\}$.

Theorem 3 Let $G$ be a graph on $n$ vertices, the Laplacian spectrum of $\bar{G}$ is:

$$
\mu_{i}(\bar{G})=n-\mu_{n-i}(G), 1 \leq i \leq n-1
$$

Corollary 1 Let $G$ be a graph on $n$ vertices, we have $\mu_{1}(G) \leq n$ with equality if and only if $\bar{G}$ is a non-connected graph.

Theorem 4 [2] A complete graph is determined by its Laplacian spectrum.

Definition 1 [5] A graph $G$ is strongly regular with parameters $n, k, \alpha, \gamma$ if 
- $G$ is not the complete graph or the graph without edges

- $G$ is k-regular

- Every two adjacent vertices have exactly $\alpha$ common neighbors

- Every two non-adjacent vertices have exactly $\gamma$ common neighbors

Theorem 5 [5] A regular connected graph is strongly regular if and only if it has exactly three distinct adjacency eigenvalues.

A strongly regular non-connected graph is the disjoint union of $r$ complete graphs $K_{k+1}$ for a given $r$.

Theorem 6 [5] Let $G$ be a connected strongly regular graph with parameters $n, k, \alpha, \gamma$ and let $k, \theta, \tau$ the eigenvalues of its adjacency matrix. Then:

$$
\begin{aligned}
& \theta=\frac{\alpha-\gamma+\sqrt{\Delta}}{2} \\
& \tau=\frac{\alpha-\gamma-\sqrt{\Delta}}{2}
\end{aligned}
$$

where

$$
\Delta=(\alpha-\gamma)^{2}+4(k-\gamma)=(\theta-\tau)^{2}
$$

Moreover, let $m_{\theta}$ (resp. $m_{\tau}$ ) the multiplicity of $\theta$ (resp. $\tau$ ), then:

$$
\begin{gathered}
m_{\theta}=-\frac{(n-1) \tau+k}{\theta-\tau} \\
m_{\tau}=\frac{(n-1) \theta+k}{\theta-\tau}
\end{gathered}
$$

That is:

$$
\begin{aligned}
& m_{\theta}=\frac{1}{2}\left(n-1-\frac{2 k+(n-1)(\alpha-\gamma)}{\sqrt{\Delta}}\right) \\
& m_{\tau}=\frac{1}{2}\left(n-1+\frac{2 k+(n-1)(\alpha-\gamma)}{\sqrt{\Delta}}\right)
\end{aligned}
$$

\section{Disjoint union of complete graphs}

The aim of this section is to show that if we consider graphs without isolated vertex then the disjoint union of complete graphs is determined by its Laplacian spectrum.

We first state some results about disjoint union of complete graphs (including isolated vertices).

Proposition 1 The Laplacian spectrum of a graph $G$ with one and only one positive Laplacian eigenvalue $a$ is $\left\{a^{(r a-r)}, 0^{(r+p)}\right\}$ and $G$ is isomorphic to $\underbrace{K_{a} \cup K_{a} \cdots \cup K_{a}}_{r \text { times }} \cup \underbrace{K_{1} \cup K_{1} \cup \cdots \cup K_{1}}_{p \text { times }}$. 
Proof : $\quad$ Let $G$ be a graph with one and only one positive Laplacian eigenvalue $a$ and let $H$ be a connected component of $G$ different from $K_{1}$. The graph $H$ has one and only one positive eigenvalue $a$. If $H$ is not a complete graph, then by theorem 2 we have $a \leq \min \{d(v), v \in V(G)\} \leq \max \{d(v), v \in$ $V(G)\}<a$, contradiction. As a result $H$ is a complete graph and $H$ is isomorphic to $K_{a}$ and there exists $r \in \mathbb{N}^{*}, p \in \mathbb{N}$ such that $G$ is isomorphic to $\underbrace{K_{a} \cup K_{a} \cdots \cup K_{a}}_{r \text { times }} \cup \underbrace{K_{1} \cup K_{1} \cup \cdots \cup K_{1}}_{p \text { times }}$.

Theorem 7 There is no cospectral non-isomorphic disjoint union of complete graphs.

ProOF : Let $G=K_{k_{1}} \cup \cdots \cup K_{k_{n}}$ and $G^{\prime}=K_{k_{1}^{\prime}} \cup \cdots \cup K_{k_{n^{\prime}}}$, we have $n=n^{\prime}$ (same number of connected components). If $G$ and $G^{\prime}$ are not isomorphic then there exists $\lambda \in \mathbb{N} \backslash\{0,1\}$ such that the number of connected components of $G$ isomorphic to $K_{\lambda}$ is different from the number of connected components of $G^{\prime}$ isomorphic to $K_{\lambda}$. Therefore, the multiplicity of $\lambda$ as an eigenvalue of the Laplacian spectrum of $G$ is different from the multiplicity of $\lambda$ as an eigenvalue of the Laplacian spectrum of $G^{\prime}$ and so $G$ and $G^{\prime}$ are not cospectral.

Theorem 8 Let $G$ be a graph without isolated vertex. If the Laplacian spectrum of $G$ is $\left\{k_{1}^{\left(k_{1}-1\right)}, k_{2}^{\left(k_{2}-1\right)}, \ldots, k_{n}^{\left(k_{n}-1\right)}, 0^{(n)}\right\}$ with $k_{i} \in \mathbb{N} \backslash\{0,1\}$ then $G$ is a disjoint union of complete graphs of order $k_{1}, \ldots, k_{n}$.

Proof : The graph $G$ has $n$ connected components (Theorem 1) $G_{1}, \ldots, G_{n}$ of order $l_{1}, \ldots, l_{n}$. We denote by $N$ the number of vertices of $G$. We have

$$
N=\sum_{i=1}^{n} l_{i}=\sum_{i=1}^{n} k_{i}
$$

Let $k_{j}$ be an eigenvalue of $G$, there exists $i$ such that $k_{j}$ is an eigenvalue of $G_{i}$, so $l_{i} \geq k_{j}$ (Theorem 22).

Let $G_{i}$ be a connected component, as $G$ does not have isolated vertices we have $l_{i}>1$ and $G_{i}$ possesses at least one eigenvalue different from 0 , let $k_{j}$ be this eigenvalue, we have $l_{i} \geq k_{j}$.

As a result

$$
\begin{aligned}
& \forall j \exists i: l_{i} \geq k_{j} \\
& \forall i \exists j: l_{i} \geq k_{j}
\end{aligned}
$$

We assume $k_{1} \geq k_{2} \geq \ldots \geq k_{n}>0$ and $l_{1} \geq l_{2} \geq \ldots \geq l_{n}>1$. We now show by induction on $j$ that $k_{n-j} \leq l_{n-j}, \forall j=0 \ldots n-1$.

- $j=0$ : we know that there exists $j$ such that $k_{j} \leq l_{n}$, so $k_{n} \leq l_{n}$.

- Let $j_{0}>0$. We assume that $\forall j<j_{0}, k_{n-j} \leq l_{n-j}$ et let us show that $k_{n-j_{0}} \leq l_{n-j_{0}}$ by contradiction. If $k_{n-j_{0}}>l_{n-j_{0}}$ then $k_{n-j_{0}}>l_{n-j}, \forall j<j_{0}$ so $k_{n-j}, j \geq j_{0}$, cannot be an eigenvalue of $G_{n-j}, j<j_{0}$. So

$$
\bigcup_{j<j_{0}}\left(S p\left(G_{n-j}\right) \backslash\{0\}\right) \subset \bigcup_{j<j_{0}}\left\{k_{n-j}^{\left(k_{n-j}-1\right)}\right\} .
$$


But

$$
\left|\bigcup_{j<j_{0}} S p\left(G_{n-j}\right) \backslash\{0\}\right|=\sum_{j<j_{0}} l_{n-j}-j_{0} \geq \sum_{j<j_{0}} k_{n-j}-j_{0}=\left|\bigcup_{j<j_{0}}\left\{k_{n-j}^{\left(k_{n-j}-1\right)}\right\}\right|
$$

so

$$
\bigcup_{j<j_{0}}\left(S p\left(G_{n-j}\right) \backslash\{0\}\right)=\bigcup_{j<j_{0}}\left\{k_{n-j}^{\left(k_{n-j}-1\right)}\right\} .
$$

As a result $k_{n-j}, j<j_{0}$ cannot be an eigenvalue of $G_{n-j_{0}}$ and as $k_{n-j_{0}}>l_{n-j_{0}}$, $k_{n-j}, j \geq j_{0}$ cannot be an eigenvalue of $G_{n-j_{0}}$. That implies that $G_{n-j_{0}}$ does not have any positive eigenvalue which contradicts that $G$ is without isolated vertex. So $k_{n-j_{0}} \leq l_{n-j_{0}}$ which conclude this induction.

As $\sum l_{i}=\sum k_{i}$ and $\forall j=1, \cdots, n, l_{j} \geq k_{j}$ we have $\forall j=1, \cdots, n, l_{j}=k_{j}$.

We now show by induction on $j$ that $S p\left(G_{n-j}\right) \backslash\{0\}=\left\{k_{n-j}^{\left(k_{n-j}-1\right)}\right\}, \forall j=$ $0, \cdots, n-1$.

- $j=0$. Let $k_{r}$ be an eigenvalue of $G_{n}$ then $k_{r} \leq l_{n}=k_{n}$ and as $k_{r} \geq k_{n}$ we have $k_{r}=k_{n}$. So the $k_{n}-1$ positive eigenvalues of $G_{n}$ are the $k_{n}$ 's.

- Let $j_{0}>0$. We assume that $\forall j<j_{0} S p\left(G_{n-j}\right) \backslash\{0\}=\left\{k_{n-j}^{\left(k_{n-j}-1\right)}\right\}$. Then $k_{n-j}^{\left(k_{n-j}-1\right)}$ for $j<j_{0}$ are not eigenvalues of $G_{n-j_{0}}$ and as $k_{n-j}=l_{n-j} \geq l_{n-j_{0}}$ for $j \geq j_{0}$ the positive eigenvalues of $G_{n-j_{0}}$ are necessarily $l_{n-j_{0}}$ i.e. $k_{n-j_{0}}^{\left(k_{n-j_{0}}-1\right)}$.

By Theorem 1 we have that $G_{i}, i=1, \cdots, n$ are the complete graphs on $k_{i}$ vertices.

\section{Disjoint union of two complete graphs}

In this section we consider the disjoint union of two complete graphs and we want to replace the condition "without isolated vertex" of Theorem 8 (this condition cannot be deduced from the spectrum) by a condition on the eigenvalues.

The spectrum of $K_{a} \cup K_{b}, a \geq b>1$ is $\left\{a^{(a-1)}, b^{(b-1)}, 0^{(2)}\right\}$. The disjoint union of two complete graphs is not in general determined by its spectrum, here is a counter-example. The Laplacian spectrum of the line graph of $K_{6}$ (which is a strongly regular graph with parameters $15,8,4,4)$ is $\left\{10^{(9)}, 6^{(5)}, 0\right\}$, so the Laplacian spectrum of $\mathcal{L}\left(K_{6}\right) \cup K_{1}$ is $\left\{10^{(9)}, 6^{(5)}, 0^{(2)}\right\}$ which is also the spectrum of $K_{10} \cup K_{6}$.

As the disjoint union $K_{a} \cup K_{a} \cup \ldots \cup K_{a}$ is determined by its spectrum 2], we assume $a \neq b$ The aim of this section is to show that a graph with Laplacian spectrum $\left\{a^{(a-1)}, b^{(b-1)}, 0^{(2)}\right\}$ with $a>\frac{5}{3} b$ is the disjoint union of two complete graphs.

The paper 3] and the thesis [1] study graphs with few eigenvalues. We can in particular mention the following results:

Theorem 9 [3, Theorem 2.1 and Corollary 2.4] A k-regular connected graph with exactly two positive Laplacian eigenvalues $a$ and $b$ is strongly regular with parameters $n, k, \alpha, \gamma$ with $\gamma=\frac{a b}{n}$. Moreover $k$ verifies $k^{2}-k(a+b-1)-\gamma+\gamma n=$ 0 . 
Remark 1 In [3], the relation $\gamma=\frac{a b}{n}$ and the equation $k^{2}-k(a+b-1)-\gamma+\gamma n=$ 0 are given in the proof of Theorem 2.1.

Theorem 10 [3] Let $G$ be a non-regular graph with Laplacian spectrum $\left\{a^{(a-1)}, b^{(b-1)}, 0\right\}$ with $a>b>1, a, b \in \mathbb{N}^{*}$. Then $G$ possesses exactly two different degrees $k_{1}$ and $k_{2}\left(k_{1} \geq k_{2}\right)$ verifying: $\left\{\begin{array}{c}k_{1}+k_{2}=a+b-1 \\ k_{1} k_{2}=a b-\frac{a b}{n}\end{array}\right.$ and $k_{2} \geq b$ with $k_{2}=b$ if and only if $G$ or $\bar{G}$ is not connected.

Lemma 1 A regular graph with Laplacian spectrum $\left\{a^{(a-1)}, b^{(b-1)}, 0\right\}$ is a strongly regular graph with parameters $n, \frac{n+1}{2}, \frac{n+1}{4}, \frac{n+1}{4}$.

Moreover we have $(a-b)^{2}=a+b$.

Proof : Let $G$ be a regular graph with Laplacian spectrum $\left\{a^{(a-1)}, b^{(b-1)}, 0\right\}$, then according to Theorem $9 G$ is strongly regular with parameters $n, k, \alpha, \gamma$. The spectrum of the adjacency matrix of $G$ is $\left\{(k-a)^{(a-1)},(k-b)^{(b-1)}, k\right\}$. By Theorem [6] we have $k-b=\frac{\alpha-\gamma+\sqrt{\Delta}}{2}$ and $k-a=\frac{\alpha-\gamma-\sqrt{\Delta}}{2}$ where $\Delta=$ $(\alpha-\gamma)^{2}+4(k-\gamma)=(a-b)^{2}$ and we have $\alpha-\gamma=2 k-a-b$ so (remind that $a+b-1=n)$ :

$$
\alpha-\gamma=2 k-n-1
$$

Moreover Theorem [6 gives $b-1=\frac{1}{2}\left(n-1-\frac{2 k+(n-1)(\alpha-\gamma)}{\sqrt{\Delta}}\right)$ and $a-1=\frac{1}{2}\left(n-1+\frac{2 k+(n-1)(\alpha-\gamma)}{\sqrt{\Delta}}\right)$ so $a-b=\frac{2 k+(n-1)(\alpha-\gamma)}{\sqrt{\Delta}}$ and so

$$
(a-b)^{2}=2 k+(n-1)(\alpha-\gamma)
$$

But $a b=\gamma n$ (Theorem 9) so

$$
(a-b)^{2}=2 k+n \alpha-a b-(\alpha-\gamma)
$$

Equations 1 and 3 give:

$$
(a-b)^{2}=1+n(\alpha+1)-a b
$$

As the mean of the degrees is $k$, we have $k=\frac{2|E|}{n}$ and $2|E|$ is the sum of the Laplacian eigenvalues, so $k=\frac{a(a-1)+b(b-1)}{n}$ i.e.

$$
n k=a^{2}+b^{2}-n-1
$$

Equation 4 gives $a^{2}+b^{2}-a b-n-1=n \alpha$, using Equation 5 we have $n k-a b=n \alpha$ i.e. $n(k-\alpha)=a b$. But $a b=\gamma n$ so

$$
\alpha+\gamma=k
$$

Using $\Delta=(\alpha-\gamma)^{2}+4(k-\gamma)$ and $\Delta=(a-b)^{2}=2 k+(n-1)(\alpha-\gamma)$ (Equation 2) we obtain $(\alpha-\gamma)^{2}+4(k-\gamma)=2 k+(n-1)(\alpha-\gamma)$ but $n-1=-\alpha+\gamma+2 k-2$ (Equation 1) and $2 k=2 \alpha+2 \gamma\left(\right.$ Equation 6), so $(\alpha-\gamma)^{2}+4 \alpha=2 \alpha+2 \gamma+(\alpha+$ $3 \gamma-2)(\alpha-\gamma)$ that is

$$
(\alpha-\gamma)(4-4 \gamma)=0
$$

As a result we have $\alpha=\gamma$ or $\gamma=1$. Let us show that $\gamma=1$ is impossible: $\gamma=1$ implies $\alpha=k-1$ and Equation 1 becomes $n=k+1$ and so $G$ is the 
complete graph with $n$ vertices, which is impossible because $\left\{a^{(a-1)}, b^{(b-1)}, 0\right\}$ with $a>b>1$ is not the spectrum of a complete graph.

Finally $\alpha=\gamma=\frac{k}{2}$ (Equation 6) and (Equation (1) $k=\frac{n+1}{2}$ and (Equation 2) $(a-b)^{2}=2 k=n+1=a+b$.

Theorem 11 Let $G$ be a graph whose Laplacian spectrum is $\left\{a^{(a-1)}, b^{(b-1)}, 0\right\}$ with $a>b>1, a, b \in \mathbb{N} \backslash\{0,1\}$ and $a>\frac{5}{3} b$. Then $G$ is not regular.

Proof : Proof by contradiction. Let $G$ be a graph whose Laplacian spectrum

is $\left\{a^{(a-1)}, b^{(b-1)}, 0\right\}$ with $a>b>1, a, b \in \mathbb{N} \backslash\{0,1\}$ and $a>\frac{5}{3} b$ and we assume that $G$ is a $k$-regular graph. Then by the previous lemma we have that $G$ is strongly regular and $(a-b)^{2}=a+b$.

This implies $a^{2}-2 a b-a=b-b^{2}<0$ so $a-2 b-1<0$ and $a \leq 2 b$. Then $3 b \geq a+b=(a-b)^{2}>\frac{4}{9} b^{2}$ so $b\left(\frac{4}{9} b-3\right)<0$ which gives $b \leq 6$. We have $\frac{5}{3} b<a \leq 2 b$ and $b \leq 6$, by listing the different cases we show that $(a-b)^{2} \neq a+b$ for $b \neq 3$ :

\begin{tabular}{|c|c|c|c|}
\hline$b$ & $a$ & $a+b$ & $(a-b)^{2}$ \\
\hline \hline 3 & 6 & 9 & 9 \\
\hline \multirow{2}{*}{4} & 7 & 11 & 9 \\
\cline { 2 - 4 } & 8 & 12 & 16 \\
\hline \multirow{2}{*}{5} & 9 & 14 & 16 \\
\cline { 2 - 4 } & 10 & 15 & 25 \\
\hline \multirow{2}{*}{6} & 11 & 17 & 25 \\
\cline { 2 - 4 } & 12 & 18 & 36 \\
\hline
\end{tabular}

If $b=3$ then $a=6$ and $n+1=a+b=9$ but, according to Lemma 1, $n+1$ is even, a contradiction.

Remark 2 When $b<a \leq \frac{5}{3} b$, the equation $(a-b)^{2}=a+b$ admits an infinity of solutions; it is not difficult to show that the couples $(a, b)=\left(u_{i}, u_{i-1}\right)$ where $u_{0}=3$ and $u_{i}=u_{i-1}+i+2$ are solutions.

Lemma 2 There is no graph with Laplacian spectrum $\left\{a^{(a-1)}, b^{(b-1)}, 0\right\}$ with $a, b \in \mathbb{N} \backslash\{0,1\}$ and $a>2 b$.

Proof : Let $G$ be a graph with Laplacian spectrum $\left\{a^{(a-1)}, b^{(b-1)}, 0\right\}$ with $a, b \in \mathbb{N} \backslash\{0,1\}$ and $a>2 b$, then $G$ is not regular (Theorem[11) and by Theorem 10 we have that $G$ possesses exactly two different degrees $k_{1}$ and $k_{2}$ verifying:

$$
\left\{\begin{array}{c}
k_{1}+k_{2}=a+b-1 \\
k_{1} k_{2}=a b-\frac{a b}{n}
\end{array}\right.
$$

with $k_{2} \geq b+1$ because $G$ and $\bar{G}$ are connected $(\bar{G}$ is disconnected if and only if the greatest eigenvalue of $G$ is $|G|$, but here $|G|=a+b-1 \neq a)$.

We have $\frac{a b}{n} \in \mathbb{N}^{*}$, but $a b \neq n$ because $a+b=n+1$ and $a, b \geq 2 \Rightarrow a b \geq a+b$. So $\frac{a b}{n} \geq 2$. 
The integers $k_{1}$ and $k_{2}$ are solutions of the equation $x^{2}-(a+b-1) x+a b-\frac{a b}{n}=0$ whose discriminant is $\Delta=(a+b-1)^{2}-4 a b+4 \frac{a b}{n}$ and so $k_{1}=\frac{a+b-1+\sqrt{\Delta}}{2}, k_{2}=$ $\frac{a+b-1-\sqrt{\Delta}}{2}$.

On one hand we have:

$$
\begin{aligned}
\Delta=(a+b)^{2}-2(a+b)+1-4 a b+4 \frac{a b}{n} & =(a-b)^{2}-2(a+b)+1+4 \frac{a b}{n} \\
& \geq(a-b)^{2}-2(a+b)+9
\end{aligned}
$$

and on the other hand we have (remind that $k_{2} \geq b+1$ ):

$$
\begin{aligned}
\Delta=\left(a+b-1-2 k_{2}\right)^{2} & \leq(a+b-1-2(b+1))^{2} \\
& \leq(a-b-3)^{2}=(a-b)^{2}-6 a+6 b+9 \\
& =(a-b)^{2}-2 a-4 a+6 b+9 \text { but } a>2 b \text { i.e. }-4 a<-8 b \\
& <(a-b)^{2}-2 a-2 b+9=(a-b)^{2}-2(a+b)+9
\end{aligned}
$$

Contradiction.

Theorem 12 There is no graph with Laplacian spectrum $\left\{a^{(a-1)}, b^{(b-1)}, 0\right\}$ with $a, b \in \mathbb{N} \backslash\{0,1\}$ and $\frac{5}{3} b<a$.

Proof : Let $G$ be a graph with Laplacian spectrum $\left\{a^{(a-1)}, b^{(b-1)}, 0\right\}$ with $a, b \in \mathbb{N} \backslash\{0,1\}$ and $\frac{5}{3} b<a$. By Lemma 2 we can assume $a \leq 2 b$. The graph $G$ is not regular (Theorem 11) and by Theorem 10 we have that $G$ possesses exactly two different degrees $k_{1}$ and $k_{2}$ verifying:

$$
\left\{\begin{array}{c}
k_{1}+k_{2}=a+b-1 \\
k_{1} k_{2}=a b-\frac{a b}{n}
\end{array}\right.
$$

with $k_{2} \geq b+1$.

First we show that we have $b \geq 6$; for that aim we use the relation $\frac{5}{3} b<a \leq$ $2 b$ and list the possible values of $a$ if $b<6$ and we show that $n$ does not divide $a b$. This is summed up into the following table.

\begin{tabular}{|c|c|c|c|c|}
\hline$b$ & $a$ & $n$ & $a b$ & Does $n$ divide $a b ?$ \\
\hline \hline 3 & 6 & 8 & 18 & no \\
\hline \multirow{2}{*}{4} & 7 & 10 & 28 & no \\
\cline { 2 - 5 } & 8 & 11 & 32 & no \\
\hline \multirow{2}{*}{5} & 9 & 13 & 45 & no \\
\cline { 2 - 5 } & 10 & 14 & 50 & no \\
\hline
\end{tabular}

Henceforth we assume $b \geq 6$. Let us show that the case $k_{2}=b+1$ is impossible. If $k_{2}=b+1$ then $k_{1}=a-2$. We denote by $n_{1}$ (resp. $n_{2}$ ) the number of vertices of degree $k_{1}$ (resp. $k_{2}$ ). The sum of the degrees is on one hand $k_{1} n_{1}+k_{2} n_{2}$ and on the other hand $a(a-1)+b(b-1)$ (sum of the eigenvalues) i.e. $k_{1}\left(k_{1}+3\right)+k_{2}\left(k_{2}-3\right)+4$. So $k_{1} n_{1}+k_{2} n_{2}=k_{1}\left(k_{1}+3\right)+k_{2}\left(k_{2}-3\right)+4$ i.e. $k_{1}\left(n_{1}-k_{1}-3\right)+k_{2}\left(n_{2}-k_{2}+3\right)=4$. But $\left(n_{1}-k_{1}-3\right)+\left(n_{2}-k_{2}+3\right)=0$ because $n=k_{1}+k_{2}=n_{1}+n_{2}$ so $\left(n_{1}-k_{1}-3\right)\left(k_{1}-k_{2}\right)=4$ i.e. $\left(n_{1}-k_{1}-3\right)(a-b-3)=4$. As a result $a-b-3$ divides 4 . 
- If $a-b-3=1$ then $a=b+4$ but $a>\frac{5}{3} b=b+\frac{2}{3} b \geq b+4$ (because $b \geq 6$ ). This case is impossible.

- If $a-b-3=2$ then $a=b+5$ and $a>\frac{5}{3} b=b+\frac{2}{3} b \geq b+5$ as soon as $b \geq 8$.

- If $b=6$ then $a=11$ and $n=16, a b=66$ and 16 does not divide 66 . This case is impossible.

- If $b=7$ then $a=12$ and $n=18, a b=84$ and 18 does not divide 84 . This case is impossible.

- If $a-b-3=4$ then $a=b+7$ and $a>\frac{5}{3} b=b+\frac{2}{3} b \geq b+7$ as soon as $b \geq 11$. The cases $6 \leq b \leq 10$ are considered in the following table:

\begin{tabular}{|c|c|c|c|c|}
\hline$b$ & $a$ & $n$ & $a b$ & Does $n$ divide $a b ?$ \\
\hline \hline 6 & 13 & 18 & 78 & no \\
\hline 7 & 14 & 20 & 98 & no \\
\hline 8 & 15 & 22 & 120 & no \\
\hline 9 & 16 & 24 & 144 & yes \\
\hline 10 & 17 & 26 & 170 & no \\
\hline
\end{tabular}

For the case $b=9, a=16$ we have $k_{1}=14$ and $k_{2}=10, k_{1} k_{2}=140$ and $a b-\frac{a b}{n}=138$, this case is impossible.

As a result we have $k_{2} \geq b+2$.

We have that $k_{1}$ and $k_{2}$ are solutions of the equation $x^{2}-(a+b-1) x+a b-$ $\frac{a b}{n}=0$ whose discriminant is $\Delta=(a+b-1)^{2}-4 a b+4 \frac{a b}{n}$.

Let $\bar{d}$ be the mean degree of $G$, we have $\bar{d}=\frac{a(a-1)+b(b-1)}{n}=\frac{-2 a b+(a+b)(a+b-1)}{n}=$ $-2 \frac{a b}{n}+a+b$ so $\frac{a b}{n}=\frac{1}{2}(b+a-\bar{d}) \geq 4$ because $b \geq 6$ and $a>k_{1}>\bar{d}$ gives $a-\bar{d} \geq 2$.

We have on one hand:

$$
\begin{aligned}
\Delta=(a+b)^{2}-2(a+b)+1-4 a b+4 \frac{a b}{n} & =(a-b)^{2}-2(a+b)+1+4 \frac{a b}{n} \\
& \geq(a-b)^{2}-2(a+b)+17
\end{aligned}
$$

and on the other hand (remind that $a+b-1-2 k_{2}=n-2 k_{2}>0$ ):

$$
\begin{aligned}
\Delta=\left(a+b-1-2 k_{2}\right)^{2} & \leq(a+b-1-2(b+2))^{2} \\
& \leq(a-b-5)^{2}=(a-b)^{2}-10 a+10 b+25 \\
& =(a-b)^{2}-2(a+b)-8 a+12 b+25 \text { but }-8 a<-\frac{40}{3} b \\
& <(a-b)^{2}-2(a+b)-\frac{4}{3} b+25 \leq(a-b)^{2}-2(a+b)+17 .
\end{aligned}
$$

Contradiction.

Remark 3 If $b<a<\frac{5}{3} b$ then the system $\left\{\begin{array}{c}k_{1}+k_{2}=a+b-1 \\ k_{1} k_{2}=a b-\frac{a b}{n}\end{array}\right.$ with $k_{1}, k_{2}, a, b, n \in$ $\mathbb{N}^{*}$ admits solutions. If $(a-b)^{2}=a+b$ then $\Delta=1$ and the system admits $a$ solution if $a+b$ is even. The following table shows solutions with $a, b \leq 1000$ and $(a-b)^{2} \neq a+b$. 


\begin{tabular}{|c|c|c|c|c|}
\hline$a$ & $b$ & $\sqrt{\Delta}$ & $k_{1}$ & $k_{2}$ \\
\hline 51 & 35 & 13 & 49 & 36 \\
81 & 64 & 12 & 78 & 66 \\
190 & 153 & 32 & 187 & 155 \\
290 & 204 & 83 & 288 & 205 \\
260 & 222 & 31 & 256 & 225 \\
469 & 403 & 59 & 465 & 406 \\
595 & 528 & 58 & 590 & 532 \\
784 & 638 & 141 & 781 & 640 \\
936 & 833 & 94 & 931 & 837 \\
\hline
\end{tabular}

Theorem 13 The graph $K_{a} \cup K_{b}$ with $a, b \in \mathbb{N} \backslash\{0,1\}$ and $\frac{5}{3} b<a$ is determined by its Laplacian spectrum.

Proof : Let $G$ be a graph with Laplacian spectrum $\left\{a^{(a-1)}, b^{(b-1)}, 0^{(2)}\right\}$ with $a, b \in \mathbb{N}^{*}$ and $\frac{5}{3} b<a$ then $G$ has two connected components. If $G$ has an isolated vertex then the Laplacian spectrum of a connected component of $G$ is $\left\{a^{(a-1)}, b^{(b-1)}, 0\right\}$, which is impossible (Theorem 12). Consequently $G$ does not have isolated vertex and we apply Theorem 8

The following corollary is straightfoward thanks to Theorem 3 ;

Corollary 2 The complete bipartite graph $K_{a, b}$ with $a, b \in \mathbb{N} \backslash\{0,1\}$ and $\frac{5}{3} b<a$ is determined by its Laplacian spectrum.

\section{References}

[1] E.R. van Dam. Graphs with few eigenvalues-An interplay between combinatorics and algebra. PhD thesis, Tilburg University, 1996.

[2] E.R. van Dam and W.H. Haemers. Which graphs are determined by their spectrum? Linear Algebra and its Applications 373:241-272, 2003.

[3] E.R. van Dam and W.H. Haemers. Graphs with constant $\mu$ and $\bar{\mu}$. Discrete Mathematics 182(1-3):293-307, 1998.

[4] M. Fiedler. Algebraic connectivity of graphs. Czechoslovak Mathematical Journal, 23(98):298-305, 1973.

[5] C. Godsil and G. Royle. Algebraic Graph Theory. Springer, 2001.

[6] B. Mohar. The Laplacian Spectrum of Graphs. Graph Theory, Combinatorics, and Applications, 2:871-898, 1991. 